\title{
Calisto y las consecuencias del deseo. Una posible influencia de las Metamorphosis en la Tragicomedia
}

\author{
Cristina Guardiola-Griffiths \\ Universidad de Delaware
}

La elaboración del Calisto de la Celestina se ha fijado en ciertos aspectos de su individualidad y egoísmo. ${ }^{1}$ Calisto ha sido acusado de ser un amante cortés maltrecho, que pobremente ha aprendido las lecciones de Andreas Capellanus (Deyermond, Text-book... 218-21). También se le ha tachado de paródico (Martin 71-134). Ensimismado por su apetito desordenado se aísla de su mundo social, olvidadas las demandas de la honra y la moral (Lida de Malkiel 347-53). Bien se pudiera decir, entonces, que Calisto se rige por un deseo sexual que transgrede las normas sociales y morales de su época. Conocidos la exhortación y el propósito moral que nos ofrece Rojas en su libro, no resultaría nada extraño si hubiese tomado el parangón de Calisto de otro texto moralizado para enfatizar esa exhortación y ese propósito moral. El presente artículo ofrece una hipótesis sobre el desarrollo de este personaje y su motivación transgresora, intentando establecer una posible conexión que relacione al Calisto de la Celestina con Calisto del Ovidius moralizatus.

Los preliminares de la Celestina, como sugiere McPheeters, son a primera vista "documentos [que parecen] poco inspirados... convencionales y sin mucha relación» con la historia de Rojas (20). Pero como nos comenta el crítico inglés, tanto la carta como el prólogo enfatizan el propósito moral y didáctico de la obra. Al haberse escrito después de la primera y segunda redacción, se podría argüir que estos preliminares refuerzan la intención del libro, que pudiera haber sido algo olvidada con la entusiasta acogida de los amores ilícitos de Calisto y Melibea. Para que los jóvenes enamorados resistan "con defensivas armas» al amor, Rojas describe en la Tragicomedia sus efectos malignos. Este tono moral hace posible una conexión entre la Tragicomedia y las Metamorfosis de Ovidio, moralizadas en el siglo XIV por Pierre Berçuire y nuevamente «trasladadas» al castellano

1. Quisiera agradecer a la Universidad de Delaware su generosa beca GUR, que me facilitó la investigación necesaria para el presente artículo. 
en el siglo xv por Alfonso de Zamora. La versión moral de Zamora alegoriza las Metamorfosis, y en el segundo libro se narra la historia de la ninfa Calixto y la transformación que sufre por su encuentro amoroso. ${ }^{2}$

La presencia de Ovidio (43 B.C.- 18 A.D.) apareció lentamente en los centros de estudio y enseñanza de la Edad Media. Como menciona Schevill, aunque se hubiesen conocido sus obras, difícilmente se hubiesen leído en un ambiente dominado por "that virile spirit of the young Christian society» (6). Era la materia pagana una de las principales razones por las que hubo oposición a la lectura de Ovidio, pero tanto el poeta como su obra paulatinamente se rehabilitan a lo largo de la Edad Media. El sulmonese se convierte en protagonista de vitae que le caracterizan como cristiano y sus obras se alegorizan para interpretarlas de forma mayormente didáctica. Para los siglos XII y XIII, llamados por Traube aetas ovidiana, vemos aumentar el uso de textos ovidianos en clases de gramática y retórica, donde se leían como textos ejemplares (Brancaforte xvii). Schevill dice que se leía a Ovidio en estos siglos por el provecho derivado de su lenguaje, por el material mitólogico del cual se sacaban tramas u otros elementos narrativos, y por los preceptos amatorios de sus obras (15). La interpretación alegórica enfatizó la lección moral de la mitología ovidiana, y su popularidad continuó durante varios siglos (Schevill 17). Seguramente es la mención a tantos divinos dioses y comisión de tantos 'malignos' hechos lo que impulsa la traducida y comentada obra de Pierre Berçuire (ca. 1290-1362), el Ovidius moralizatus. Es esta última obra la que nos concierne ahora, ya que las Metamorfosis de Ovidio se conocían principalmente en comentarios moralizados como el de Berçuire (Carr, "Introduction», Text 1).

El Ovidius moralizatus formaba parte de la obra enciclopédica llamada Reductorium morale, cuyo propósito didáctico servía a los predicadores en la composición de sus sermones. No obstante, el décimoquinto texto del Reductorium morale disfrutó de una circulación mayor (Carr, "Introduction", Text 1). El comentario de Berçuire se escribe a mediados del siglo XIV, y goza de una gran popularidad hasta bien entrado el siglo Xvi. Posiblemente fue el comentario de Berçuire el que el Marqués de Santillana

2. Anteriormente ya aparecen partes de las Metamorfosis en la General Estoria de Alfonso el Sabio, donde se alaba profusamente al poeta sulmonese. El episodio de Calisto, al cual aludimos en este presente artículo, también se encuentra en la primera parte de la General Estoria. Aunque cabe la posibilidad de que Rojas conociera la versión alfonsina, nos parece que más probablemente fuese el posible parangón la traducida versión de Berçuire, ya que el contenido es mayormente moralizado y comparte ciertas características geográficas y temporales con La Celestina.

Curiosamente, hemos encontrado una historia particular de Calistone en un manuscrito con letra de mediados del siglo xv: la Historia de la infanta Callisto o Parrasis, hija de Licaón; se halla entre los folios 70r y 72v, BNM 5644 de la Biblioteca Nacional de Madrid. La Historia se describe en Philobiblon, BETA MANID 3275 (URL: http://sunsite.berkeley.edu/Philobiblon/ BETA/3275.html). 
pidió traducir al castellano. El marqués menciona la versión del texto ovidiano traducido en la carta que escribe a su hijo, Pedro González, estando éste en Salamanca.

A ruego e instançia mía, primero que de otro alguno, se han vulgarizado en este reyno algunos poetas, assi como la Eneida de Virgilio, el Libro mayor de las transformaçiones de Ovidio, las Tragedias de Lucio Anio Séneca e muchas otras cosas en que yo me he deleytado fasta este tiempo. (456-57)

Por lo tanto, el terminus ante quem de la obra se debe fijar en el año 1452, ya que el hijo del marqués termina sus estudios en tal fecha. Como ha averiguado Carr, el texto de Berçuire probablemente fue traducido por Alfonso de Zamora, bachiller en decretos y traductor del marqués ("Introduction", Text 4-5). Como no se conoce otra versión cuatrocentista de las Metamorfosis, es posible que corresponda al Libro mayor de las Transformaciones que alude Santillana. Esto, a su vez, hace posible que se pudiera acceder al texto moralizado en Castilla ya desde mediados del siglo XV. Según Gilman, las Metamorfosis formaban parte de la biblioteca de otro bachiller, el autor de la Celestina, pero duda que fueran escritas en latín (43233). ¿Sería acaso posible que Rojas, medio siglo después, conociera y utilizara esta versión de las Transformaciones en la composición de su obra?

Dada esta posibilidad, nos centraremos en la traducción de Zamora, obra que también enfatiza un propósito moral:
Conviene a saber que muchas uezes las fablas por figuras \& exenplifficaciones se deuen usar por que dende algund moral entendemiento sea sacado... Liçita cosa es que si el omne pudiere delas espinas coja uuas \& que miel dela piedra chupe \& quel azeyte del muy duro guijaro para el tome... Asi es que enesta mi presente obreta que de aqueste mi uolumen por titulo quiero que sea non en- tiendo si non de tarde en tarde tañer el entendimiento literal delas fablas mas sola mente çerca de la exposición moral \& la allegorica trabajar siguiendo conuiene saber el libro de ouidio que se dize methamorfoseos (1-2)

No hay duda en la intención moralizante de Zamora, aunque quizás sí exprese un desdén por la obra que traduce. Las fábulas de los poetas paganos, como las espinas, la piedra, o el guijarro, por poco provecho que tengan, no se deben desechar. Estas fábulas tienen su intención moral si se interpretan como figuras o ejemplos. Por tal razón, Zamora decide explicar las metamorfosis, sacando de ellas las cualidades mayormente docentes. 
El libro segundo de las Metamorfosis, que Zamora traduce escuetamente, cuenta la transformación de una joven Calisto, llamada en esta versión Calistone. En la metamorfosis la doncella sale de caza, en cuya actividad la encuentra el dios Júpiter. Ansioso de poseerla, Júpiter se disfraza de mujer y se le acerca, y así disfrazado logra su deseo. Cuando Juno descubre el adulterio se venga de Calistone, transformándola en osa. Varios años después ésta se encuentra con Arcadio, hijo suyo ya mayor y fruto de su violación. Éste intenta matarla, pero un Júpiter compadecido evita la muerte de Calistone haciendo de madre e hijo estrellas.

Aunque la versión trate de varias metamorfosis (Júpiter se convierte en mujer; Calistone y Arcadio en constelaciones), la metamorfosis que mayormente resalta es la de Calistone en osa. Su transformación se explica de tal forma que implica su culpabilidad. Esa culpabilidad la interpreta Zamora de la siguiente manera:

$\&$ asi en verdat acaesçe que algún omne o alguna persona faz forniçio conel diablo por pecado \& del conçibe \& dolor pare \& maldat ella por cierto de fenbra en ossa se muda. (72-73)

La traducción de Zamora sólo implica lo que narra el comentario de Berçuire: la metamorfosis hace que la violación de Calistone sea su propia culpa, al buscar un momento de descanso después de la caza. Su descanso se convierte en un "forniçio conel diablo por pecado", ya que se interpreta como una manera de seducir al dios Júpiter. Así interpretado, el descanso se transforma de un acontecimiento pasivo en uno activo. La metamorfosis de Calistone ocurre no tanto por la venganza de una mujer desdeñada, sino porque la ninfa quiso pecar como mujer adúltera.

Lo transgresivo de esta acción se comprueba en la transformación de Calistone en osa. Es corroborada en los bestiarios, que describen al oso como animal cuya vitalidad reside en sus brazos y órganos reproductores:

The bear is said to get its name because the female shapes her new-born cub with her mouth, ore, giving it, so to speak, its beginning, orsus.... It is born only thirty days after conception, and as a result of this rapid fertility it is born unformed.

The bear's head is not strong; its greatest strength lies in its arms and loins; for this reason bears sometimes stand upright. (Aberdeen Bestiary 15r)

Según Shipley, el uso de los bestiarios en la Celestina se ajusta a una función pervertida, y en la obra los animales "are inverted once again... with the animality the Bestiary tradition had refined away» (23). Aunque Shipley no trata específicamente de este caso, su análisis es sugerente 
para el presente artículo. Respecto a la osa, se ve que da nombre a la especie, cuya fertilidad se exagera al describir un parto de corto plazo. Este parto relámpago se implica en el nacimiento de Arcadio, ya que ni siquiera se narra. La descripción de los órganos de mayor y menor utilidad para el oso hace pensar en un animal no tan inteligente como fuerte. La cabeza es más bien débil, mientras que los brazos y las partes pudendas tienen mayor potencia. Así descritos, los órganos que más importancia tienen son los que se asocian con la agresividad y lo sexual —órganos de cierta importancia en la Tragicomedia. El deseo sexual de Calisto se ve en numerosos momentos de la obra, y es en los momentos de actividad (o deseada actividad) cuando vemos aludidos los brazos. Dice del cordón de Melibea:

$\bigcirc$ mezquino de mí, que assaz bien me fuera del cielo otorgado que de mis braços fueras hecho y texido y no de seda como eres, porque ellos gozaran cada día de rodear y ceñir con devida reverencia aquellos miembros que tú, sin sentir ni gozar de la gloria, siempre tienes abraçados. ¿○ qué secretos avrás visto de aquella excellente ymagen? $(187)^{3}$

El cordón de Melibea, imaginado como brazos de Calisto, no podría sino sugerir un erotismo en el que se ven aludidas las partes anatómicas arriba mencionadas. A esta cita podríamos añadir la del primer encuentro amoroso: "O angélica ymagen, o preciosa perla, ante quien el mundo es feo. $\bigcirc$ mi señora y mi gloria, en mis brazos te tengo y no lo creo. Mora en mi persona tanta turbación de plazer que me haze no sentir todo el gozo que poseo» (284). Y también se ve aludido en las palabras de Lucrecia, ya pasado casi un mes de gozo: "Ya me duele a mí la cabeza descuchar y no a ellos de hablar ni los braços de retoçar ni las bocas de besar» (324). La descripción de un Calisto que disfruta y posee con sus brazos y otros miembros hace posible su comparación con la Calistone de las Metamorfosis.

Ciertas semejanzas de índole ambiental unen al Calisto de la Tragicomedia con la Calistone de las Metamorfosis. La descripción de una Calistone cazadora la sitúa en un lugar aislado, que además la asocia con un comportamiento masculino. Ella: "[e]ra una donzella mucho fermosa en un monte onde caçaua ca caçadora era \& deziasse calistone fija de licaon» (72). Esta introducción enfatiza ciertas cualidades que más se asocian con el hombre que con la mujer. ${ }^{4}$ Aunque dice que es hermosa, no describe su

3. Utilizamos la edición de Severin, por ser tan asequible como amena. No obstante, hacemos también mención a la excelente edición de Lacarra Lanz, cuyos aportes al mundo social y el discurso médico de La Celestina son de inestimable valor.

4. Aludo aquí por primera vez a la obvia diferencia de género que separa un Calisto de la otra. Falta por profundizar en esta diferencia genérica; baste ahora decir que la caracterización 
belleza tanto como su actividad y linaje. Es cazadora y es noble, como la mención de Licaón nos sugiere. Esta introducción nos induce a una comparación con Calisto. El argumento de la obra lo llama «de noble linage... de stado medio," mientras que el argumento del primer auto añade lo siguiente: «Entrando Calisto en una huerta en pos dun halcón suyo, hallo y a Melibea, de cuyo amor preso, començóle a hablar...» (85). La referencia al halcón indica tanto su condición como el ejercicio en el que plausiblemente se encuentra: la caza.

El neblí perdido de Calisto le lleva a la huerta de Melibea, un espacio que, aunque localizado dentro de los confines de lo urbano, no comparte características urbanas. La huerta de Melibea, protegida por sus altas paredes, nos ofrece otro punto de comparación con la metamorfosis ovidiana, ya que se separa del espacio urbano y se transforma a un espacio bucólico. Es en este espacio natural donde los amantes, a causa del ejercicio de la caza, se encuentran y dan muestras de su amor.

Tanto la actividad como el lugar de ambos Calistos conduce a pensar en el topos de la caza de amor. La caza como actividad simbólica del encuentro amoroso nos lleva a ver cómo ambos Calistos caen «presos» en amores. La manifestación del amor como prisión metafórica, elaborada en tantísimos poemas cancioneriles y quizás nunca mejor vista que en la ficción sentimental la Cárcel de amor, se ve en ambos Calistos, ya que el uno se aprisiona por el amor que siente y la otra es aprisionada por el amor de Júpiter. Se pudiera diferenciar a los Calistos en este momento, ya que el uno seduce a Melibea y la otra, desde un punto de vista moderno y compasivo, es seducida y víctima de una violación. Desde esta perspectiva, bien se podría hablar de una prisión al ser violada, no sólo durante el momento amoroso sino también durante los meses de embarazo que siguen. Pero como hemos visto, en vez de ser víctima del comportamiento lujurioso del dios Júpiter, el texto moralizado cuestiona esa interpretación moderna. El momento de ocio que toma Calistone mientras descansa de la caza se ve como un pecado diabólico, cometido por una amante transgresora y sexual, cuyos delitos motivan su propia metamorfosis. Así interpretado, el texto moralizado ofrece otro punto de comparación con la Tragicomedia. El autor de la Tragicomedia no duda en explicar los efectos malignos que conlleva el amor, principalmente a través de Calisto.

Los estudios de Wack han examinado el punto de vista médico sobre la enfermedad del amor, frecuentemente un punto de vista reñido con el religioso. Las teorías médicas del medioevo explican la facilidad con la que se amolda Calisto a ser víctima del amor hereos. Como joven noble y

masculina de la ninfa ovidiana hace posible a primera vista una comparación con Calisto. No obstante, el uso de una mujer como modelo del protagonista rojano enfatiza su conducta desmesuradamente apasionada, conducta que poco se relaciona con las virtudes consideradas masculinas durante la Edad Media. 
ocioso cae víctima de amor, pero según un contexto moral su enfermedad le convierte en "loco enamorado". El desordenado apetito de Calisto y las consecuencias de su amor resultan ser tan heréticos como diabólicos. Como consecuencia de ese apetito busca a Celestina, cuyo poder de hechicera se menciona dos veces en el primer acto. Severin ha demostrado la importancia de la palabra hechicera, y aunque nos haya ilustrado sobre las diferencias entre la magia blanca y la magia negra y lo que cada una conlleva, el temor que exhiben los criados de Calisto nos ofrece testimonio del poder diabólico de la alcahueta (Severin, Witchcraft, 7-8). El conjuro que hace sobre el hilado al final del tercer acto da prueba de ello, como también la muerte que llega a todos quienes tienen contacto con ese hilado (Deyermond, Cordón... 6-12).

Las semejanzas morales de ambos textos y las semejanzas ambientales y físicas de ambos Calistos constituyen un primer punto de comparación. En lo que sigue del presente artículo veremos cómo la interpretación alegórica de Calistone en las Metamorfosis se pudiera comparar con el comportamiento de Calisto en La Celestina. El texto de las Metamorfosis interpreta así la transformación de la joven ninfa:

\& asi en verdat acaesçe que algún omne o alguna persona faz forniçio conel diablo por pecado \& del conçibe \& dolor pare \& maldat ella por cierto de fenbra en ossa se muda. por quanto la humana figura que son las Razonables costunbres \& condiçiones dexa \& las de osso bestiales \& carnales condiçiones ujste $\&$ entonces cobra pies coruos que es corua \& peruersa entinçion \& piel de osso que es ujl \& infame conuersacion $\&$ boz horrible que es torpe \& non honesta fabla que quando alguno se faze pecador todas las buenas costunbres se mudan enel que son la afeçion. la fabla. \& la conuersaçion. (72-73)

La interpretación de esta metamorfosis nos haría pensar que el hombre transformado en oso es el hombre vencido por «bestiales \& carnales condiciones.» El amor del que padece Calisto ha sido descrito por Martin como el de "a man overcome by lust... whose increasing mental ineptitude can perhaps be said to find a culmination in his death when his brains are literally scattered - over the walls and street around Melibea's garden» (106-7). La humana figura de Calisto, cada vez más dominado por sus pasiones y menos capaz de distinguir entre el bien y el mal, llega a olvidarse de las razonables costumbres y condiciones.

La pérdida de buenas costumbres se explica a través del pecado, que se especifica en la metamorfosis moralizada como un deseo erótico que convierte al hombre en bestia. A la transformación literal de Calistone se le atribuye cierta simbología, lo cual, si se le aplica al Calisto de la Tragicomedia, describe perfectamente su creciente pasión por Melibea. El ena- 
morarse locamente de Melibea causa en Calisto la mudanza de buenas costumbres en malas, las cuales se manifiestan a través de la intención y afición perversa con la cual persigue a Melibea, la deshonesta habla con la que se refiere a ella, y la vil e infame conversación que con ella mantiene. La piel de osa entendida como "corua \& peruersa entinçion» se puede entrever muy pronto en la obra. En la primera conversación entre Sempronio y Calisto, éste ensalza a Melibea, haciendo de ella un dios y de él un humilde servidor: "Por dios la creo, por dios la confesso, y no creo que hay otro soberano en el cielo aunque entre nosotros mora» (95). Calisto se presenta como amante cortés, que desdeña su propia persona y la salvación de su espíritu para ensalzar a la dama. No obstante, Sempronio destapa un significado carnal y de cuestionable moral en las palabras altisonantes de su amo:

Sempronio: Ríome, que no pensava que havía peor invención de peccado que en Sodoma.

Calisto: ¿Cómo?

Sempronio: Porque aquellos procuraron abbominable uso con los ángeles no conoscidos, y tú con el que confiessas ser Dios.

Calisto: ¡Maldito seas! Que hecho me has reýr, lo que no pensé ogaño. (95)

Sempronio entiende que el amor de Calisto por Melibea no es sublimado ni inocente. Refiriéndose a los acontecimientos bíblicos de Sodoma, Sempronio entiende que la hipérbole sagrada de Calisto no indica otra cosa que un deseo lascivo. Su interpretación se corrobora en la risa de Calisto, cuya simpatía por el sirviente misógino aumenta con la promesa de llevar a Melibea "hasta la cama». La comicidad de esta escena encubre un contenido serio y prohibido que, aunque provocante a risa, implica la deshonra de Melibea (Gargano 296).

La intención torcida y perversa de Calisto se enfatiza más en el intento de alcanzar a Melibea. Calisto no repara en las intenciones ni en las consecuencias de su pasión. Su actitud hipócrita ya demuestra una falta de razonar.

Calisto: ¿Cómo as pensado de hazer esta piedad?

Sempronio: Yo te lo diré. Días ha grandes que conozco en fin desta vezindad una vieja barbuda que se dize Celestina, hechizera, astuta, sagaz en quantas maldades hay. Entiendo que passan de cinco mil virgos los que se han hecho y desecho por su autoridad en esta cibdad. A las duras peñas promeverá y provocará a luxuria, si quiere. (103) 
La propuesta de Sempronio se describe como obra de piedad, pero poco tiene de piadosa. La descripción de Celestina no pudiera ser más tajante, ya que contrasta con la de otros prudentes intercesores de la ficción sentimental. Celestina es de aspecto físico desagradable, con parecidos en cuanto a su condición y cualidad. Su parte en esta propuesta es la de proveer a la doncella, para que Calisto cumpla sus deseos con ella sin que nada se descubra. Esta descripción subraya la parodia del amor cortesano de Calisto, puesto que su reacción descubre un malogrado disfraz de lujuria (Lacarra 148). Calisto no recela de la descripción de Celestina ni de su parte en la propuesta. Inmediatamente quiere hablar con la alcahueta, muy a despecho de un Pármeno que todavía le es fiel. Las amonestaciones de Pármeno son en vano, aunque no podrían ser más enfáticas. Recalca el carácter negativo de Celestina e intenta aconsejar a Calisto que deje el servicio de la alcahueta y que emplee su dinero en mejores medios:

Pármeno: Digo, señor, que yrían mejor empleadas tus franquezas en presentes y servicios a Melibea, que no dar dineros a aquella que yo conozco [...] Señor, porque perderse el otro día el neblí fue causa de tu entrada en la huerta de Melibea a le buscar; la entrada causa de la veer y hablar; la habla engendró amor; el amor parió tu pena; la pena causará perder tu cuerpo y el alma y hazienda. Y lo que más dello siento es venir a manos de aquella trotaconventos, después de tres vezes emplumada.

Calisto: ¡Assí, Pármeno, di más desso, que me agrada! Pues mejor me parece quanto más la desalavas; cumpla conmigo y enplúmenla la quarta; dessentido eres; sin pena hablas; no te duele donde a mí, Pármeno. (134-35)

Las palabras de Pármeno son bastante reveladoras, y narran los acontecimientos que llevaron al enamoramiento de Calisto. El amor de Calisto "parió» el dolor penoso que ahora siente (palabras que, aunque pudieran ser puramente casuales, matizan la metamorfosis de ambos Calistos). Pero el Calisto de Rojas no sigue el consejo de Pármeno, ni presta atención al sirviente salvo cuando dice algo que le interese. La referencia a una Celestina emplumada, con el castigo de untar con miel y plumas a la alcahueta, marca uno de los oficios de Celestina, del cual pretende beneficiarse Calisto.

Si el oficio de alcahueta no hace vacilar a Calisto de que deje sus objetivos, las sugeridas acusaciones de hechicería tampoco lo detienen. Ni los fieles reproches de Pármeno, que insinúan la maldad de la alcahueta, ni la cicatriz que lleva Celestina en la cara, posible signo diabólico menciona- 
do a principios del cuarto acto por Lucrecia, serviría de acicate a Calisto para entender el carácter maligno de la alcahueta (Severin, Celestina 15253). A todos estos avisos y señales Calisto hace caso omiso, prefiriendo poner en efecto sus corruptos deseos. Es más, la complicidad de Calisto en el enamoramiento demoníaco de Melibea se deduce de las palabras que dice Celestina sobre el cordón: "Calla y no fatigues, que más aguda es la lima que yo tengo que fuerte essa cadena que te atormenta; yo la cortaré con ella por que tú quedes suelto. Por ende dame licencia, que es muy tarde, y déjame llevar el cordón, porque como sabes, tengo dél necessidad» (191). Las palabras "como sabes», añadidas a la Tragicomedia subrayan el conocimiento que Calisto tendría sobre el uso del cordón. Implican que Calisto pudiera ser consciente de la philocaptio, y por lo tanto, le acusan de ser un agente perverso en la corrupción de Melibea.

A la vez que se entiende la perversa intención de Calisto cuando habla con los sirvientes, la deshonesta y torpe habla que se interpreta por la voz horrible del oso se ve en las conversaciones que mantiene con Melibea. Ejemplos tenemos de ello a partir del primer acto. Como Deyermond ha mencionado, la primera escena de la Tragicomedia se basa en una lección mal aprendida del De amore de Andreas Capellanus. En el lenguaje exagerado e inapropiado que Calisto utiliza con Melibea se descubre una rudeza que ella describe como torpe, y cuyo significado puede ser entendido como lascivo o impúdico (Lacarra 144). Melibea señala la importancia de utilizar las palabras apropiadas no solo en su reacción violenta a la primera conversación con Calisto, sino también en la primera embajada de Celestina, intermediaria y portavoz del enamorado. Melibea enfatiza la necesidad de expresarse a través de otras palabras que estén fuera de sospecha, lo que sugiere su comprensión (y complicidad) en la deshonesta y lujuriosa habla de Calisto. El significado del dolor de muelas, la petición de que regrese Celestina «muy secretamente» y su preocupación de ser mal entendida por Calisto (por "cruel o arrebatada o deshonesta») podrían presuponer una Melibea consciente del dolor que sufre Calisto y consentidora de un futuro trato con él. Su afán de querer hablar con determinadas palabras, por lo tanto, implica su conocimiento de la imprudente torpeza que mana del habla de Calisto.

Los crudos deseos de Calisto se desbordan en habla cada vez más grosera una vez conseguido el acceso al huerto. Reflejado en las conversaciones del huerto, abunda un desbordado interés en lo sexual. Eufemismos sexuales corrientes en la poesía cancioneril también abundan en estos encuentros. Calisto se disculpa de tocar la ropa de su señora con desvergonzadas manos, ya que jamás se pensó digno de merecerla, pero no por eso cambia su comportamiento. Quisiera tener «testigos de su gloria» en los ojos de Lucrecia, quien luego verá con mal disimulada envidia el proseguir de los amores. Este hablar cortés de Melibea mantiene la apariencia de cortesía sin reparar en lo transgresor de los hechos. No obstan- 
te, el querer alargar Melibea el juego amoroso con palabras delicadas y cortesanas choca con la actitud más grosera de Calisto. La famosa frase, "Quien primero quiere comer ave, primero ha de quitarle las plumas» (324), dista mucho de los servicios, sacrificios, devoción y obras pías que primero tenía ofrecidos. Cualquier intento de mantener su cortés habla ha desaparecido con estas palabras tan explícitas.

Las constantes protestas de Melibea nos hacen ver el objetivo de Calisto: un trato amoroso que pudiera ser entendido por la piel de oso, explicada como vil e infame conversación. El uso de este vocablo por amancebamiento, o trato ilícito con mujer, se entiende en la misma obra, al mandar Melibea que Calisto sosiegue y deje el «enojoso vso y conuersación incomportable» de las manos. Calisto, sin embargo, piensa en el trato noble: "Jamás querría, señora, que amaneciesse, según la gloria y descanso que mi sentido recibe de la noble conuersación de tus delicados miembros» (324). No llega a entender la falta moral que comete ni cuando su conversación intolerable toca a su honra. Las muertes de Sempronio y Pármeno, causadas por sus trámites con Celestina, indiscutiblemente repercutirían en su condición social. Su reacción evoca la infamia y vileza que ha llegado a su estado. Poco es el lamento que hace Calisto ante la pérdida de Sempronio y Pármeno, y poco es el cuidado en lo que atañe a su nombre, que habría sufrido desdoro con la ejecución pública de sus sirvientes. Poco le cuesta desentenderse del problema con algún fingimiento para poder seguir su trato ilícito. Los sirvientes, que antes habían sido descritos con tanto afecto, ahora son calumniados.

[Sempronio y Pármeno] eran sobrados y esforçados, agora o en otro tiempo de pagar havían. La vieja era mala y falsa, según paresce que hazía trato con ellos, y assí que riñeron sobre la capa del justo. Permissión fue divina que assí acabassen en pago de muchos adulterios que por su intercessión o causa son cometidos. (281-82)

Quienes antes habían sido alabados por su sabiduría, lealtad y virtud, ahora son "sobrados», malos y falsos. La hipocresía de Calisto es evidente, ya que critica la parte que ellos tenían en el negocio de Melibea, sin caer en cuenta de la suya. Del mismo modo que se le pudiera llamar hipócrita por su deshonesto comportamiento, también se le podría tachar de vil e infame por su cobardía. Decide no comportarse como amo y evitar el problema causado por las tres muertes. «Pues por más mal y daño que me venga, no dexaré de complir el mandado de aquella por quien todo esto se ha causado. Que más me va en conseguir la ganancia de la gloria que spero, que en la pérdida de morir los que murieron» (281). Calisto es consciente de su deber, pero prefiere dedicarse a la consecución de goces mayores. Decide fingirse ausente o loco y proseguir con los amores ya alcanzados de Melibea. Este comportamiento, corroborado en sucesivos 
encuentros, lleva al lector a verle según la interpretación moralizada de Ovidio.

Ambas historias, así interpretadas, se podrían entender como una transformación basada en la trasgresión sexual de esa persona. Para la Calisto ovidiana, esa transformación se describe explícitamente en el comentario traducido de Berçuire. El texto de Berçuire interpreta el relato ovidiano, dando a cada paso una explicación moralizante. Estas explicaciones hacen que se pueda ver más estrechamente al Calisto de La Celestina utilizando las mismas fuentes que la Calistone de la Metamorfosis moralizada. Según la interpretación de Berçuire, quienquiera que peque y cometa "fornicio con el diablo» se convierte en oso. Esta conversión se describe minuciosamente, y la transformación de cada parte corporal tiene una explicación moralizante. Así vemos que la transformación de Calisto sirve como punto de partida para analizar el comportamiento del Calisto castellano. El deseo que siente Calisto le transforma en una especie de bestia, que ya no razona sino que actúa según sus instintos amorosos. Por tales razones, es posible ver en el Calisto moralizado de las Metamorfosis un paradigma para el Calisto de Rojas.

\section{Bibliografía}

Aberdeen Bestiary. Ed. Iain Beavan. 24 October 2003 (URL: http://www. clues.abdn.ac.uk:8080/seek_idx.html.

BETA: Bibliografia Española de Textos Antiguos. Comp. Charles B. Faulhaber, Angel Gomez Moreno, Angela Moll, and Antonio Cortijo. URL: http://sunsite.berkeley.edu/Philobiblon/phhmbe.html. Vol. 2002, n. 2 (July 2002). In: PhiloBiblon (URL: http://sunsite.berkeley.edu/Philobiblon/phhm.htm

BRANCAFORTE, Benito, ed. Las Metamorfosis y las Heroidas de Ovidio en la General Estoria de Alfonso el Sabio. Madison, WI: Hispanic Seminary of Medieval Studies, 1990.

CARR, Derek C. «La transformación de las Transformaciones: El caso de los Morales de Ovidio, traducción castellana del Ovidius Moralizatus de Pierre Berçuire (Madrid, B.N., Mas. 10144, ¿ca. 1435?).» Studi filologici e letterari in memoria di Danilo Aguzzi-Barbagli. Ed. D. Boccassini. Stony Brook, NY: Forum Italicum, 1997. 12-22.

- ed. Text and concordance of Morales de Ovidio: a fifteenth-century Castilian translation of the Ovidius moralizatus (Pierre Berçuire): Madrid, Biblioteca Nacional ms. 10144. By P. Bersuire. Madison, WI: Hispanic Seminary of Medieval Studies, 1992. 
COSSÍO, José María de. Fábulas mitológicas en España. Madrid: EspasaCalpe, 1952.

DEYERMOND, Alan D. "The Text-Book Mishandled: Andreas Capellanus and the Opening Scene of La Celestina.» Neophilologus 45 (1961): 218-221.

— «Hilado-Cordón-Cadena: symbolic equivalence in La Celestina.» Celestinesca 1.1 (1977): 6-12.

GARGANO, Antonio. "¡Maldito seas! Que fecho me has reír'. Intención cómica y contenido serio en La Celestina.» La Celestina, V Centenario (1499-1999). Ed. Pedraza Jiménez, et. al. Cuenca: Ediciones de la Universidad de Castilla-La Mancha, 2001. 273-282.

GILMAN, Stephen. La España de Fernando de Rojas: panorama intelectual y social de La Celestina. Madrid: Taurus, Colección Persiles, 1974.

LACARRA, María Eugenia, ed. La Celestina. By Fernando de Rojas. $2^{\text {a }}$ ed. Madison, WI: Hispanic Seminary of Medieval Studies, 1995.

LIDA DE MALKIEL, María Rosa. La originalidad artística de La Celestina. Buenos Aires, EUDEBA, 1962.

LÓPEZ DE MENDOZA, Íñigo, Marqués de Santillana. Obras completas. Ed. Ángel Gómez Moreno y Maxim P.A.M. Kerkhof. Barcelona: Planeta, 1988.

MARTIN, June Hall. Love's Fools: Aucassin, Troilus, Calisto and the Parody of the Courtly Lover. London: Tamesis Books Ltd., 1972.

MCPHEETERS, D. W. Estudios humanísticos sobre La Celestina. Potomac, MD: Scripta Humanistica, 1985.

ROJAS, Fernando de. La Celestina. Ed. D. Severin. Madrid: Cátedra, 1992.

RUSSELL, P. E. «La magia como tema integral de la Tragicomedia de Calisto y Melibea.» Studia philologica: homenaje ofrecido a Dámaso Alonso III. Madrid: Gredos, 1963, pp. 337-354.

SEVERIN, Dorothy S. "Fernando de Rojas and Celestina: the Author's intention from Comedia to Tragicomedia de Calisto y Melibea.» Celestinesca 5.1 (1981): 1-5.

- Witchcraft in Celestina. London: Department of Hispanic Studies, Queen Mary and Westfield College, 1995.

SCHEVILL, Rudolph. Ovid and the Renascence in Spain. Hildesheim, New York: Georg Olms Verlag, 1971.

SHIPLEY, George, "Bestiary References in Fernando de Rojas's, La Celestina (1499): The Ironic Undermining of Authority,» Corónica 2.1 (1975): 22-23.

WACK, Mary Frances. «The Measure of Pleasure: Peter of Spain on Men, Women, and Lovesickness,» Viator 17 (1986): 173-196.

- Lovesickness in the Middle Ages. Philadelphia: University of Pennsylvania Press, 1990. 
Guardiola-Griffiths, Cristina, "Calisto y las consecuencias del deseo. Una posible influencia de las Metamorphosis en la Tragicomedia», Celestinesca 28 (2004), pp. 33-46.

\section{RESUMEN}

El presente artículo ofrece una hipótesis sobre el desarrollo de Calisto, intentando establecer una posible conexión entre el personaje de La Celestina y la Calisto del Ovidius moralizatus.

PAlabras Clave: La Celestina, Metamorfosis, bestiario, amor loco, transgresión.

\section{ABSTRACT}

The following article suggests a possible model for Calisto as a character in Rojas's work. It puts forth a connection between the Celestina and the Ovidius moralizatus.

KEY WORDS: La Celestina, Metamorfosis, bestiary, love's fools, transgresion.

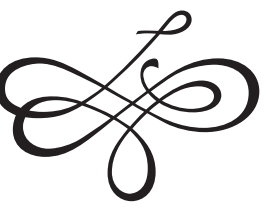

